\title{
Aromatase Inhibitor-associated Bone Loss in Breast Cancer
}

\author{
a report by \\ Peyman Hadji, MD \\ Head, Department of Endocrinology, Reproductive Medicine, and Osteoporosis, Philipps-Marburg University
}

DOI: 10.17925/EOH.2007.0.1.18

In 2006, breast cancer was the most commonly diagnosed cancer among women, comprising approximately $31 \%$ of new cancer cases, and resulting in $15 \%$ of all cancer-related deaths in the US. ${ }^{1}$ In a large proportion of the newly diagnosed breast cancer cases, breast cancer tumors are hormone-responsive; therefore, estrogen and its receptor have become the targets of choice for therapeutic intervention. Estrogen suppression prevents or reduces growth of estrogen receptor-positive $(E R+)$ tumors, and can be achieved through surgical or targeted therapeutic modalities. Early surgical and radiological ovarian ablation strategies have given way to more targeted therapies involving the detection of ER status and compounds such as tamoxifen that block tumor-cell growth at the ER level. Tamoxifen was the therapy of choice for hormone-responsive breast cancer and is still commonly prescribed. However, many patients still experience disease recurrence despite ongoing tamoxifen therapy. Furthermore, tamoxifen is associated with serious adverse events, most notably thromboembolism (blood clots) and endometrial cancer, and therapy is limited to five years. Therefore, alternative approaches with improved efficacy to target ER+ breast cancer tumors have been developed.

Aromatase inhibitors (Als) block estrogen production in the peripheral tissues by preventing the last step in estrogen biosynthesis. The superior efficacy and more favorable side-effect profile demonstrated by Als have allowed them to begin to replace tamoxifen as the adjuvant therapy of choice for post-menopausal women with ER+ breast cancer. ${ }^{2-5}$ However, one caveat of $\mathrm{Al}$ therapy is accelerated bone loss and increased fracture risk in a population of women that may already be at risk for fracture related to chemotherapy, low bone mineral density (BMD), age, history of fragility fracture after the age of 50, family history of hip fracture, or treatment history. ${ }^{4-8}$ The goal of adjuvant hormonal therapy for early breast cancer is to prevent local or distant disease recurrence or prolong disease-free survival (DFS) or overall survival (OS). Recent head-to-head clinical trials demonstrate that $\mathrm{Al}$ treatment is superior to tamoxifen for

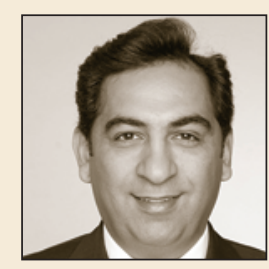

Peyman Hadji, MD, is Head of the Department of Endocrinology, Reproductive Medicine, and Osteoporosis and Professor in the Department of Obstetrics, Gynecology, and Endocrinology at Philipps-Marburg University. Professor Hadji is also Head of the Osteoporosis Section of the German Society of Gynecological Endocrinology and Reproductive Medicine, a Board Member of Dachverband Osteologie and the German Menopause Society, and a Member of the German Society of Gynecology and Obstetrics, the American Society for Bone and Mineral Research, the International Society of Clinical Densitometry, and the International Menopause Society. achieving this goal. To this end, maximum clinical benefit may be achieved by combining Al therapy with bisphosphonates in patients at risk for fracture to ensure optimal breast cancer management and prevent bone loss.9.10 This review briefly summarizes the available data regarding aromatase inhibitor-associated bone loss (AIBL) and provides insight into ongoing trials for the prevention of AIBL with bisphosphonates.

\section{Bone Loss and Fracture Risk Associated with Breast Cancer}

In general, women reach their peak BMD around age 20-30. The most significant loss occurs at menopause and subsequently slows to approximately $1 \%$ per year thereafter. ${ }^{11}$ Reduction in serum estrogen is directly responsible for bone loss and changes in the bone microarchitecture that lead to increased fracture risk. One large prospective study of women $\geq 65$ years old demonstrated a direct association between estrogen levels and fracture incidence. Women in this study who had undetectable estrogen levels had an increased risk of hip and vertebral fractures compared with women who had very low but still detectable estrogen levels. ${ }^{12}$ Thus, chemotherapy and cancer treatment regimens that either directly or indirectly reduce circulating estrogen can have a dramatic deleterious effect on bone and fracture risk.

Evidence from a variety of sources indicates that breast cancer is associated with significant changes in bone metabolism and higher incidence of fracture in both pre- and post-menopausal women. In one recent study, Kanis and colleagues assessed the impact of breast cancer on skeletal health by comparing the vertebral fracture risk in women with newly diagnosed, non-metastatic breast cancer with an age-matched control group of women without breast cancer. ${ }^{13}$ The women with breast cancer were found to have a nearly five-fold increased risk of vertebral fracture during the first three years following diagnosis, and patients with soft-tissue relapse had an approximate 23 -fold increased risk.13 Importantly, the authors note that the fracture risk may have been underestimated by at least $25 \%$ because half of the breast cancer patients were receiving the bisphosphonate clodronate. Similarly, results from the Women's Health Initiative Observational study indicate that breast cancer survivors have a $31 \%$ increased risk of fracture compared with agematched women with no cancer, even after adjustments for age, weight, ethnicity, and geographical region of enrolment. ${ }^{14}$ In one prospective study of pre-menopausal women with early breast cancer, primary chemotherapy-induced premature menopause resulted in significant decline in BMD within six months, and this decline increased through 12 months. ${ }^{15}$ Taken together, these studies clearly demonstrate that women with breast cancer are at increased risk for bone loss and fracture compared with healthy age-matched women. 
Tamoxifen therapy has been widely used as adjuvant therapy for postmenopausal women with breast cancer. One of the assumed clinical benefits of tamoxifen is the bone-protective effects in post-menopausal women. However, pre-menopausal women receiving tamoxifen experience bone loss and are at increased risk for fracture. ${ }^{16}$ Interestingly, recent data presented at the San Antonio Breast Cancer Symposium demonstrated that at least $50 \%$ of women who have completed five years of tamoxifen therapy already have pronounced bone loss, ${ }^{17}$ indicating that the bone-protective effects of tamoxifen therapy may not always be sufficient to prevent bone loss in women being treated for breast cancer.

\section{Aromatase Inhibitor-associated Bone Loss}

In post-menopausal women with hormone-receptor positive breast cancer, third-generation Als are replacing tamoxifen as the preferred adjuvant therapy. Recent large, randomized trials have demonstrated improved efficacy of Als over tamoxifen, and current guidelines now recommend Al adjuvant therapy to reduce the risk of disease recurrence. Aromatase inhibitors prevent the biosynthetic conversion of androgens to estrogens in peripheral tissues, such as breast, muscle, and adipose tissue, and thereby deplete residual estrogen in post-menopausal women. The third-generation Als letrozole, anastrozole, and exemestane are capable of inhibiting approximately $97-99 \%$ of aromatase enzyme activity, resulting in nearly complete depletion of circulating estrogen (see Table 1). ${ }^{18,19}$ In the Breast International Group (BIG) 1-98 study, the near complete depletion of estrogen by letrozole resulted in a significant $19 \%$ reduction in the risk of disease recurrence $(p=0.003)$ and a significant $27 \%$ reduction in the risk of distant recurrence $(p=0.001) .{ }^{8}$ A recent update of this study demonstrated that five years of letrozole monotherapy resulted in a significant $18 \%$ reduction $(p=0.007)$ in the risk of disease recurrence compared with tamoxifen monotherapy. ${ }^{3}$ Similarly, results from the MA-17 trial show that letrozole reduces the risk of disease recurrence (hazard ratio $(H R)=0.58, p<0.001)$ and significantly improves distant DFS (HR=0.6, $p=0.002$ ) compared with placebo. ${ }^{6}$ Furthermore, an analysis of the MA-17 trial according to receptor status found that compared with placebo, letrozole provided a significant benefit in DFS $(H R=0.49)$, distant $D F S(H R=0.53)$, and OS survival $(H R=0.58)$ in patients with $\mathrm{ER}+$ /progesterone receptor-positive $(\mathrm{PgR}+)$ tumors. ${ }^{20}$ Low estrogen levels in post-menopausal women are known to be important for maintaining bone density, and Al therapy that depletes residual estrogen can further accelerate post-menopausal bone loss. The rapid bone loss attributable to the abrupt reduction in estrogen is clearly a class effect and is associated with all Als. Therefore, all women with breast cancer undergoing long-term $\mathrm{Al}$ therapy are at risk for developing AIBL.

Although large clinical trials examining the efficacy of Al therapy have consistently shown improved DFS compared with tamoxifen, they have also demonstrated increased bone loss and fracture rates in patients receiving Als (see Figure 1).5,6,8,21 Results from the Arimidex, Tamoxifen, Alone or in Combination (ATAC) trial have shown that after two years of anastrozole treatment, BMD was significantly reduced (median of $4.1 \%$ in lumbar spine and $3.9 \%$ in hip), ${ }^{22}$ and after five years of Al therapy, patients experienced a shift in BMD from normal to osteopenic with a BMD loss of approximately $8 \%$ at the spine and hip. ${ }^{23}$ During this time, none of the patients with normal BMD at baseline and only $5 \%$ of patients who were osteopenic at baseline became osteoporotic, but
Figure 1: Aromatase Inhibitor Therapy Increases Fractures in Women with Breast Cancer ${ }^{5,6,8,21}$

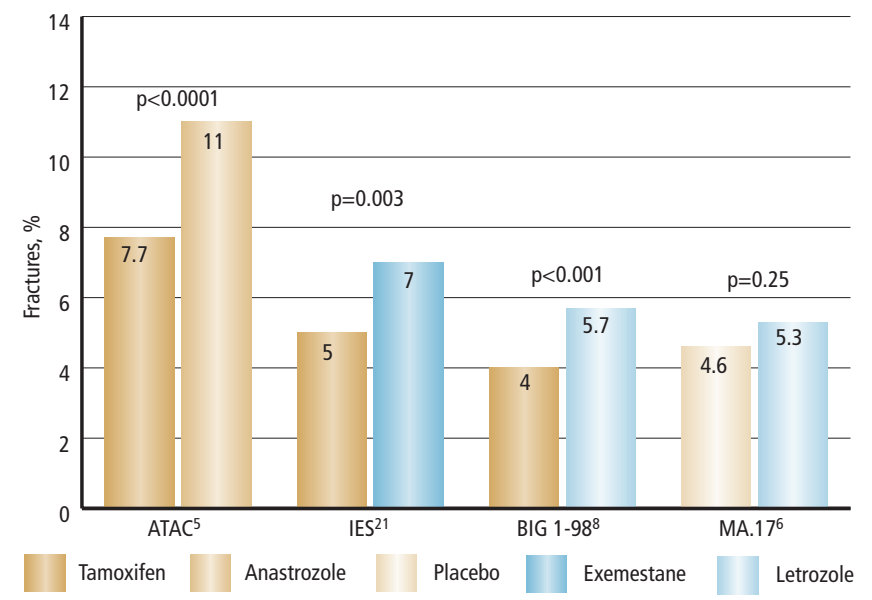

Fracture incidence in patients receiving aromatase inhibitor therapy, tamoxifen, and placebo in four clinical trials. ATAC = Arimidex, Tamoxifen, Alone or in Combination; IES = Intergroup Exemestane Study; BIG 1-98 = Breast International Group; MA.17 = National Cancer Institute of Canada Clinical Trials Group MA.17.

Figure 2: Up-front Zoledronic Acid Prevents Aromatase Inhibitor-associated Bone Loss in Post-menopausal Women with Breast Cancer
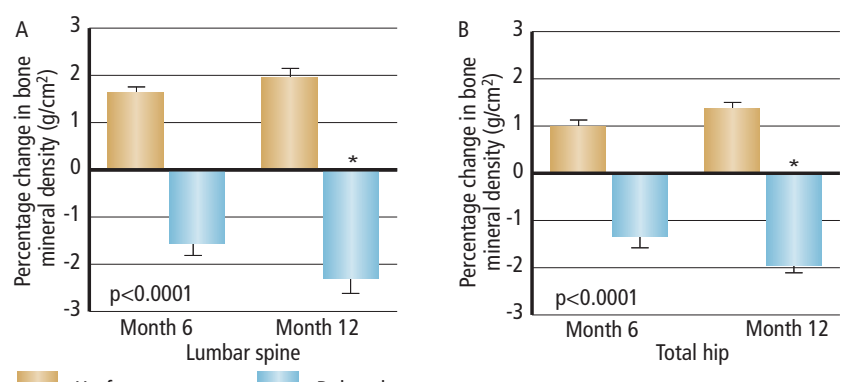

Up-front group

Delayed group

Mean (standard error of the mean) per cent change in bone mineral density of the lumbar spine and the total hip at months 6 and 12 in women with early-stage breast cancer administered upfront or delayed zoledronic acid; ${ }^{*} p$ values correspond to intragroup comparisons from baseline to month 12. Reprinted from Brufsky A, et al., ${ }^{9}$ with permission from the American Society of Clinical Oncology.

Figure 3: Up-front Zoledronic Acid Prevents Bone Loss in Pre-menopausal Women with Breast Cancer Receiving Endocrine Therapy

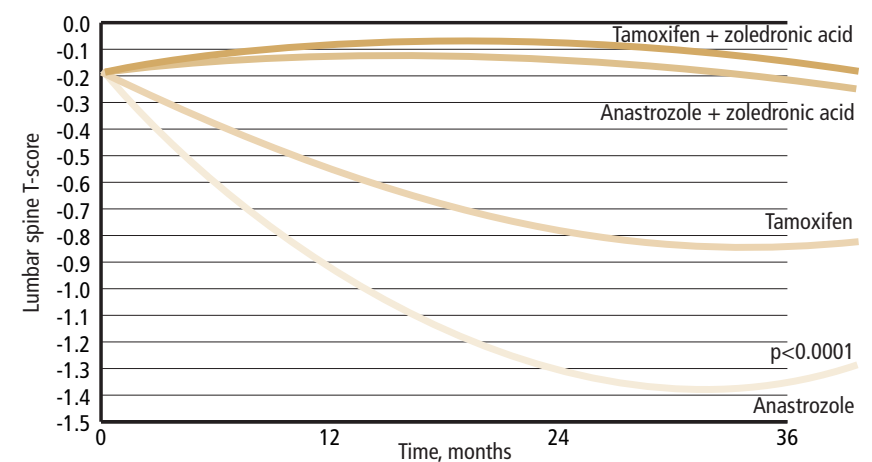

Changes from baseline T-scores over time in the lumbar spine of patients treated for 36 months with anastrozole or tamoxifen \pm zoledronic acid. All patients received goserelin. Reprinted from Gnant MF, et al., ${ }^{10}$ with permission from the American Society of Clinical Oncology. 
Breast Cancer

Table 1: Aromatase Inhibition and Depletion of Estrogen Levels

\begin{tabular}{lcr} 
Aromatase Inhibitor & Aromatase Inhibition (\%) & Plasma Estradiol \\
\hline Letrozole $^{18}$ & $>99.1$ & $12 / 12$ undetectable \\
& & Mean 2.1pmol/l \\
\hline Anastrozole $^{18}$ & 97.3 & $9 / 12$ undetectable \\
& & Mean 2.6pmol/l \\
\hline Exemestane $^{19}$ & 97.9 & $7 / 9$ undetectable \\
& & Mean 2.8pmol/l \\
\hline
\end{tabular}

Table 2: Aromatase Inhibitor Superiority to Tamoxifen

\begin{tabular}{|c|c|c|c|c|}
\hline Trial & $\begin{array}{l}\text { Median Follow-up } \\
\text { (months) }\end{array}$ & Aromatase Inhibitor & $\begin{array}{l}\text { Absolute Benefit* } \\
(\%)\end{array}$ & $\begin{array}{r}\text { Hazard } \\
\text { Ratio }\end{array}$ \\
\hline$\overline{\operatorname{ATAC}^{5}}$ & 68 & Anastrozole (up-front) & 3.7 & 0.83 \\
\hline BIG 1-98 & 26 & Letrozole (up-front) & 2.6 & 0.81 \\
\hline $\mathrm{BIG} 1-98 t^{3}$ & 51 & Letrozole (up-front) & 2.9 & 0.82 \\
\hline$\overline{\mathrm{IES}^{35}}$ & 31 & Exemestane & 4.7 & 0.68 \\
\hline$\overline{\mathrm{IES}^{4}}$ & 56 & Exemestane & 3.4 & 0.76 \\
\hline$\overline{\text { ARNO/ABCSG }}{ }^{7}$ & 28 & Anastrozole & 3.1 & 0.60 \\
\hline$\overline{\mathrm{MA}-17^{6}}$ & 30 & Letrozole (vs placebo) & 4.6 & 0.58 \\
\hline
\end{tabular}

*Disease-free survival. + Analysis restricted to monotherapy arms.

ATAC $=$ Arimidex, Tamoxifen, Alone or in Combination; BIG 1-98 = Breast International Group

1-98 collaborative group; IES = Intergroup Exemestane Study; $A R N O / A B C S G=$ Arimidex

Nolvadex 95 Study/Austrian Breast and Colorectal Cancer Study Group; MA.17 = National

Cancer Institute of Canada Clinical Trials Group MA.17.

approximately $50 \%$ of patients with normal BMD became osteopenic. ${ }^{23}$ Not only did the fracture rate increase, but fractures occurred earlier in patients treated with anastrozole, and the proportion of patients who became osteoporotic cannot explain the fracture rate. ${ }^{23}$ Thus, fracture risk may not correlate with BMD as closely as would be expected. This is consistent with the data from the National Osteoporosis Risk Assessment (NORA), which showed that $>80 \%$ of fractures occur in healthy postmenopausal women with T-scores $>-2.5$ (52\% occurring in osteopenic women). ${ }^{24}$ Analyses from the BIG 1-98, Intergroup Exemestane Study (IES), and MA-17 trials have revealed similar increases in bone loss and fracture incidence. After a median follow-up of two years in the BIG 198 study, patients receiving letrozole had significantly more frequent fractures $(p<0.001) .{ }^{8}$ However, in the MA-17 trial that compared letrozole treatment with placebo, there was a trend towards increased fractures only in the letrozole group, and patients receiving letrozole had a longer median time to experiencing a first fracture. ${ }^{6}$ Recent results from the IES show that patients who switched from tamoxifen to exemestane experienced both significant decreases $(p<0.0001)$ in lumbar spine and hip BMD within six months and a significant increase $(p=0.003)$ in fracture incidence after 24 months of follow-up. ${ }^{21}$ As a whole, data from these trials indicate that Als accelerate bone loss and increase fracture risk irrespective of whether the patient has received prior adjuvant therapy with tamoxifen. Therefore, bone management strategies are needed to maximize the clinical benefits achieved with Al therapy.

Preventing Bone Loss and Reducing Fracture Risk in Breast Cancer Patients

Given the natural decline in estrogen and increased bone loss seen in healthy post-menopausal women, preventing accelerated bone loss and reducing fracture risk in breast cancer patients receiving adjuvant $\mathrm{Al}$ therapy is of great importance. Fortunately, data from recent clinical trials indicate that the deleterious effects of Al therapy on the skeleton can be effectively managed with bisphosphonates, and clinical guidelines are beginning to address the needs of this rapidly growing population. The current American Society of Clinical Oncology (ASCO) guidelines for surveillance of bone loss in breast cancer patients rely on BMD as the primary indicator to direct treatment options. ${ }^{25}$ For patients with a normal T-score (>-1), physicians are to provide patients with lifestyle advice and recommend supplementation with calcium and vitamin $D$. In women with osteopenic BMD (T-score -1 to -2.5 ), calcium and vitamin D are again recommended, and bisphosphonate therapy should be considered if the patient has additional risk factors. Women with the most severe bone loss (T-scores $\leq-2.5$ ) should receive calcium and vitamin D supplements and begin bisphosphonate therapy with alendronate, risedronate, or zoledronic acid (ZOL). ${ }^{25}$ Likewise, although not intended to directly address the bone health of women with breast cancer, guidelines from the National Osteoporosis Foundation (NOF) ${ }^{26}$ and World Health Organization $(\mathrm{WHO})^{27}$ also hinge on $\mathrm{BMD}$ measurements, but these guidelines have begun to incorporate additional risk factors into the treatment paradigm. The NOF recommends pharmacological therapy, including bisphosphonate treatment, for patients who fall within one of the following categories: patients with a T-score $<-2$; those with a T-score $<-1.5$ with one or more risk factors; and those who have experienced a prior hip or vertebral fracture. ${ }^{26}$ The WHO osteoporosis guidelines advocate treatment for patients with osteoporosis (T-score $\leq-2.5$ ), or osteopenic patients (T-score -1 to -2.5 ) who have additional strong risk factors. ${ }^{27}$ Thus, there appears to be a growing consensus among the medical community to consider both BMD scores (when available) and additional risk factors when assessing patient risk and making treatment decisions. This trend is supported by the fact that more than half ( $52 \%)$ of fractures reported in the National Osteoporosis Risk Assessment (NORA) study occurred in healthy osteopenic women (mean age, 65 years) who had T-scores between -1 and $-2.5 .{ }^{24}$ Furthermore, it may be necessary to begin treatment for bone loss early during breast cancer therapy to minimize the deleterious effects of treatment on the skeleton and preserve patient quality of life and physical functioning. The available data indicate that the fracture risk experienced by breast cancer patients receiving Al therapy does not sufficiently correlate with BMD loss alone, and additional risk factors in conjunction with BMD measurements should be assessed to determine fracture risk.

Several recent clinical trials have directly addressed AIBL, and the results strongly indicate that intravenous bisphosphonates may be necessary to prevent fractures in post-menopausal breast cancer patients at high risk for bone loss and fracture. The Zometa ${ }^{\circledR} /$ Femara ${ }^{\circledR}$ Adjuvant Synergy Trials (Z-FAST and ZO-FAST) were designed to determine whether intravenous $\mathrm{ZOL} 4 \mathrm{mg}$ given every six months at the beginning of $\mathrm{Al}$ therapy (up-front) will provide benefit over ZOL given at the first sign of bone loss (T-score $<-2$ or fracture, i.e. delayed). ${ }^{28}$ The Z-FAST study demonstrated that up-front ZOL increased lumbar spine (1.9\%) and total hip BMD (1.3\%), while delaying ZOL resulted in a loss of BMD ($2.4 \%$ and $-1.98 \%$, respectively) after 12 months on study (see Figure 2). ${ }^{9}$ Furthermore, an integrated analysis of 12 -month data from these two studies confirms that up-front ZOL prevents bone loss during $\mathrm{Al}$ therapy and reduces the release of the biochemical markers of bone turnover. ${ }^{29}$ An additional benefit of up-front therapy was a trend towards reduced disease recurrence. ${ }^{29}$ After two years of therapy, patients who received up-front ZOL further gained BMD over the 
delayed-start group. In the up-front ZOL group, bone markers were persistently suppressed, and lumbar spine and total hip BMD increased $5.9 \%$ and $4.7 \%$ respectively, compared with the delayed group..$^{30}$ These long-term data suggest that ZOL can effectively prevent AIBL.

Pre-menopausal women with hormone receptor-positive breast cancer require ovarian ablation to achieve complete endocrine suppression and improved OS; however, this therapy may result in significant bone loss and increased fracture risk. The Austrian Breast and Colorectal Cancer Study Group (ABCSG-12) trial was designed to assess whether goserelin plus either tamoxifen or anastrozole improved OS and DFS in this patient population. ${ }^{10}$ In the BMD sub-protocol of this trial, patients received adjuvant therapy with or without intravenous ZOL $4 \mathrm{mg}$ every six months and BMD was measured by dual-energy $\mathrm{X}$-ray absorptiometry at baseline, six, 12, 24, and 36 months. Results from this subprotocol clearly

\author{
Adjuvant AI therapy in concert with \\ careful management of bone health \\ may allow patients to achieve \\ optimal therapeutic outcomes.
}

demonstrated that ZOL prevented bone loss associated with goserelin followed by either anastrozole or tamoxifen therapy in pre-menopausal women with chemotherapy-induced menopause..$^{10}$ In the absence of ZOL, there was a significant decrease in lumbar spine T-scores from baseline after three years of anastrozole (mean change $-2.6, p<0.0001$ ) and tamoxifen (mean change $-1.1, \mathrm{p}<0.0001$ ) (see Figure 3), ${ }^{10}$ with corresponding decreases in lumbar spine BMD (mean change $-17.4 \%$ and $-11.6 \%$, respectively; $\mathrm{p}<0.0001$ for both). Notably, after 36 months of adjuvant therapy, concomitant ZOL treatment maintained stable BMD in both anastrozole- and tamoxifen-treated women. Without ZOL there was a 30\% absolute increase from baseline in osteopenic patients in both the tamoxifen and anastrozole groups and a $24 \%$ absolute increase from baseline in osteoporotic patients in the anastrozole group. In the patients who received ZOL plus tamoxifen there was only a $3 \%$ increase in osteoporosis, while patients who received $\mathrm{ZOL}$ plus anastrozole experienced only a $15 \%$ increase in osteopenia, but did not develop osteoporosis. Taken together, results from these trials indicate that up-front $\mathrm{ZOL}$ can prevent bone loss associated with Al therapy in both pre- and post-menopausal women. Oral bisphosphonates have demonstrated limited efficacy in preventing bone loss associated with chemotherapy-induced menopause, and there are no published reports in the AIBL setting. Two small studies of women with breast cancer receiving clodronate ${ }^{31}$ and risedronate ${ }^{32}$ showed an increase in BMD after two years of oral bisphosphonate therapy. However, a 10-year follow-up of the adjuvant clodronate trial found that BMD loss was slowed but not prevented and did not reach statistical significance. ${ }^{33}$ Another potential drawback of oral bisphosphonate therapy is low patient compliance. In a recent analysis of healthcare claims, 57\% of patients receiving oral bisphosphonates were found to be non-compliant with therapy, which resulted in increased fracture risk compared with compliant patients. ${ }^{34}$ The lack of available efficacy data and poor compliance for oral bisphosphonates suggest that they are not a viable therapeutic choice in the AIBL setting.

\section{Discussion}

Al therapies have consistently demonstrated superior efficacy for DFS compared with tamoxifen and have increasingly become the first choice for adjuvant therapy in hormone receptor-positive breast cancer. ${ }^{5}$ In addition to reducing the risk of disease recurrence, letrozole provides significant clinical benefit in distant DFS. ${ }^{6}$ Moreover, among ER+/PgR+ patients, letrozole significantly increases OS compared with placebo. ${ }^{20} \mathrm{Al}$ therapy has a more favorable safety profile compared with tamoxifen, ${ }^{8}$ and the deleterious effects on bone can be easily managed. Given that all women are susceptible to fractures when BMD is low, it is becoming increasingly important to address the accelerated bone loss and increased fracture risk associated with Al and other cancer therapies. Evidence from several recent clinical trials indicates that up-front ZOL can prevent bone loss in both preand post-menopausal women receiving Al therapy. Therefore, bone health management guidelines may need to be updated to reflect the findings from the Z-/ZO-FAST and ABCSG-12 trials. To that end, it is now apparent that the osteoporotic T-score threshold for treatment is too low and that fracture risk assessment should take account of overall risk factors, including Al therapy and other cancer treatments, age, fracture history, corticosteroid use, and BMD, when possible. This comprehensive risk assessment can then be used to guide treatment decisions. In summary, adjuvant Al therapy in concert with careful management of bone health may allow patients to achieve optimal therapeutic outcomes in the adjuvant setting without increasing the patients' fracture risk.

\section{Acknowledgements}

Financial support for medical editorial assistance was provided by Novartis Pharmaceuticals. I thank Michael Hobert, PhD, ProEd Communications, Inc., for his medical editorial assistance with this manuscript.
1. Jemal A, et al., CA Cancer J Clin, 2006;56:106-30.

2. Buzdar AU, Breast Dis, 2005;24:107-17.

3. Coates AS, et al., J Clin Oncol, 2007;25:486-92.

4. Coombes RC, et al., Lancet, 2007;369:559-70.

5. Howell A, et al., Lancet, 2005;365:60-62.

6. Goss PE, et al., J Natl Cancer Inst, 2005;97:1262-71.

7. Jakesz R, et al., Lancet, 2005;366:455-62.

8. Thurlimann B, et al., N Engl J Med, 2005;353:2747-57.

9. Brufsky A, et al., J Clin Oncol, 2007;25:829-36.

10. Gnant MF, et al., J Clin Oncol, 2007;25:820-28.

11. Osteoporos Int, 1997;7:1-6.

12. Cummings SR, et al., N Engl J Med, 1998;339:733-8.

13. Kanis JA, Br J Cancer, 1999;79:1179-81.

14. Chen Z, et al., Arch Intern Med, 2005;165:552-8.
15. Shapiro CL, et al., J Clin Oncol, 2001;19:3306-11.

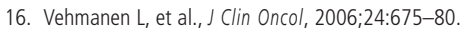

17. Whannel K, et al., Breast Cancer Res Treat, 2006;100:S187 (Abstract 4046).

18. Geisler J, et al., J Clin Oncol, 2002;20:751-7.

19. Geisler J, et al., Clin Cancer Res, 1998;4:2089-93.

20. Goss PE, et al., I Clin Oncol, 2007;25:2006-11.

21. Coleman RE, Lancet Oncol, 2007;8:119-27.

22. Eastell $R$, et al., J Bone Miner Res, 2006;21:1215-23.

23. Coleman R, J Clin Oncol, 2006;24 Suppl:5s. (Abstract 511).

24. Siris ES, et al., Arch Intern Med, 2004;164:1108-12.

25. Hillner BE, et al., J Clin Oncol, 2003;21:4042-57.

26. National Osteoporosis Foundation, Physicians Guide: Pharmacologic Options, available at: www.nof.org/physguide/phamacologic.htm. Accessed March 8, 2007.

27. World Health Organization, Prevention and Management of Osteoporosis, WHO Technical Report Series 921, 2003;1-192.

28. Aapro M, Breast, 2006;15(Suppl. 1):S30-40.

29. Brufsky A, et al., Breast Cancer Res Treat, 2006;100(Suppl. 1):S25 (Abstract 107)

30. Brufsky A, et al., Breast Cancer Res Treat, 2006;100(Suppl. 1):S233 (Abstract 5060)

31. Saarto T, et al., Br J Cancer, 1997;75:602-5.

32. Delmas PD, et al., J Clin Oncol, 1997;15:955-62.

33. Saarto T, et al., J Clin Oncol, 2006;24(Suppl.):46S (Abstract 676).

34. Siris ES, et al., Mayo Clin Proc, 2006;81:1013-22.

35. Coombes RC, et al., N Engl J Med, 2004;350:1081-92. 